PNL-2564

\title{
Projected Spent Fuel Storage Requirements
}

March 1978

Pacific Northwest Laboratory

Richland, Washington 99352

Operated for the

U.S. Department of Energy

by

$\frac{7}{2}$ 


\title{
NOTICE
}

This report was prepared as an account of work sponsored by the United States Covernment, Neither the United States nor the Department of Energy; oor any of their empioynes, nor any of their contractors. subcontractors, or their empioyees. makes any warranty, express or implied, or assumes any legai liabitity or responsibility for the accuracy, completeness or usefulness of any informaticn, apparatus, product or process disciosed, or represents that its use wouid not infringa privately owned rights.

The views, opinions and conciusions contained in this raport are those of the contractor and do not necessarily represent those of the United States Government or the United States Department of Energy.

\author{
PACIFIC NORTHWEST LABORATORY \\ operated by \\ BATTELLLF \\ for the \\ UNITED STATES DEPARTMENT OF ENERCY \\ Under Contract EY-76-C-06-1त30
}

\author{
Printed in the Lin.ted States of Americe \\ Avallabie from \\ Nationai Technical information Service \\ United Siaies Department of Commerce \\ 5285 Port Royai Road \\ Saringtield, Virginia 22757
}

Price: Prinied Ccors_.:- Microtiche $\$ 3.00$

\begin{tabular}{|c|c|}
\hline *bages & $\begin{array}{c}\text { NTIS } \\
\text { Selling Price }\end{array}$ \\
\hline $301-0,5$ & 5450 \\
\hline$y: 5-350$ & $\$ 5.00$ \\
\hline 351.073 & 55.50 \\
\hline 156.160 & 6500 \\
\hline $191-12$ & 46.50 \\
\hline $125-150$ & 5800 \\
\hline $151-1,15$ & 3775 \\
\hline $175-2.6$ & 5030 \\
\hline $201-22$ & 56.75 \\
\hline $3 a b-250$ & $\sin 6$ \\
\hline 251.25 & $\sin x$ \\
\hline $76-310$ & 572.5 \\
\hline
\end{tabular}




\title{
33679000492332
}

PROJECTED SPENT FUEL STORAGE REQUIREMENTS

\author{
by \\ A. J. Boegel \\ M. K. White \\ C. E. Willingham
}

March 1978

Pacific Northwest Laboratory

Richland, Washington 99352

Operated By

Battelle Memorial Institute 



\section{CONTENTS}

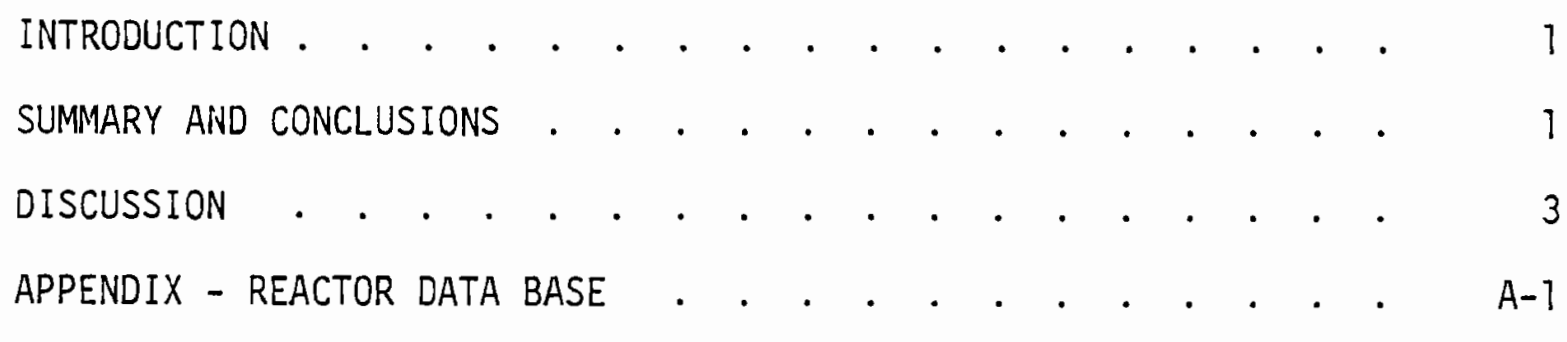




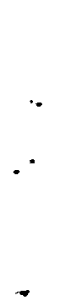




\section{PROJECTED SPENT FUEL STORAGE REQUIREMENTS}

\section{INTRODUCTION}

The amount of post reactor basin retrievable storage capacity required between now and 1990 for spent fuel depends largely on the actions taken by the nuclear industry before 1985. This date represents the earliest achievable availability of any such storage capacity other than additional water basins. The range of possible industry actions includes expansion of reactor or away from reactor (AFR) water basin capacity, discontinuing the practice of maintaining a full core reserve ( $F C R$ ) in reactor basins, and shipping fuel within a utility from a reactor with a full basin to one with excess storage capacity.

Utilities are considering taking some or all of these actions between now and 1985 to avoid reactor shutdowns due to lack of spent fuel storage capability. This analysis identifies the post-reactor basin storage capacity requirements between now and 1990 for various combinations of these utility actions in order to establish the full range of possible storage requirements.

\section{SUMMARY AND CONCLUSIONS}

Some recent analyses performed by others (NRC, DOE, etc.) $(1,2)$ have indicated that no spent fuel storage shortage will occur until after 1985. Those results are based on assumptions about the availability and usage of existing or planned storage basins belonging to General Electric, Allied General Nuclear Services, and Exxon. This analys is makes no speculation about the availability of such capacity, but instead identifies the requirements for spent fuel storage capacity which must be met by some combination of private capacity, spent fuel facility lag storage and ultimately, spent fuel storage facility capacity.

The combined actions of reracking reactor basins, transferring spent fuel within utilities, and relaxing the policy of reserving reactor basin capacity for a full core discharge are insufficient to meet pre-1985 spent 
fuel storage capacity requirements. Additional storage capacity is required before 1985 for at least 1000 BWR assemblies and 2000 PWR assemblies. Storage capacity must be provided for a minimum of 12,000 BWR assemblies and 7,000 PWR assemblies by 1990. This is depicted as the minimum spent fuel storage requirement in Figure 1 .

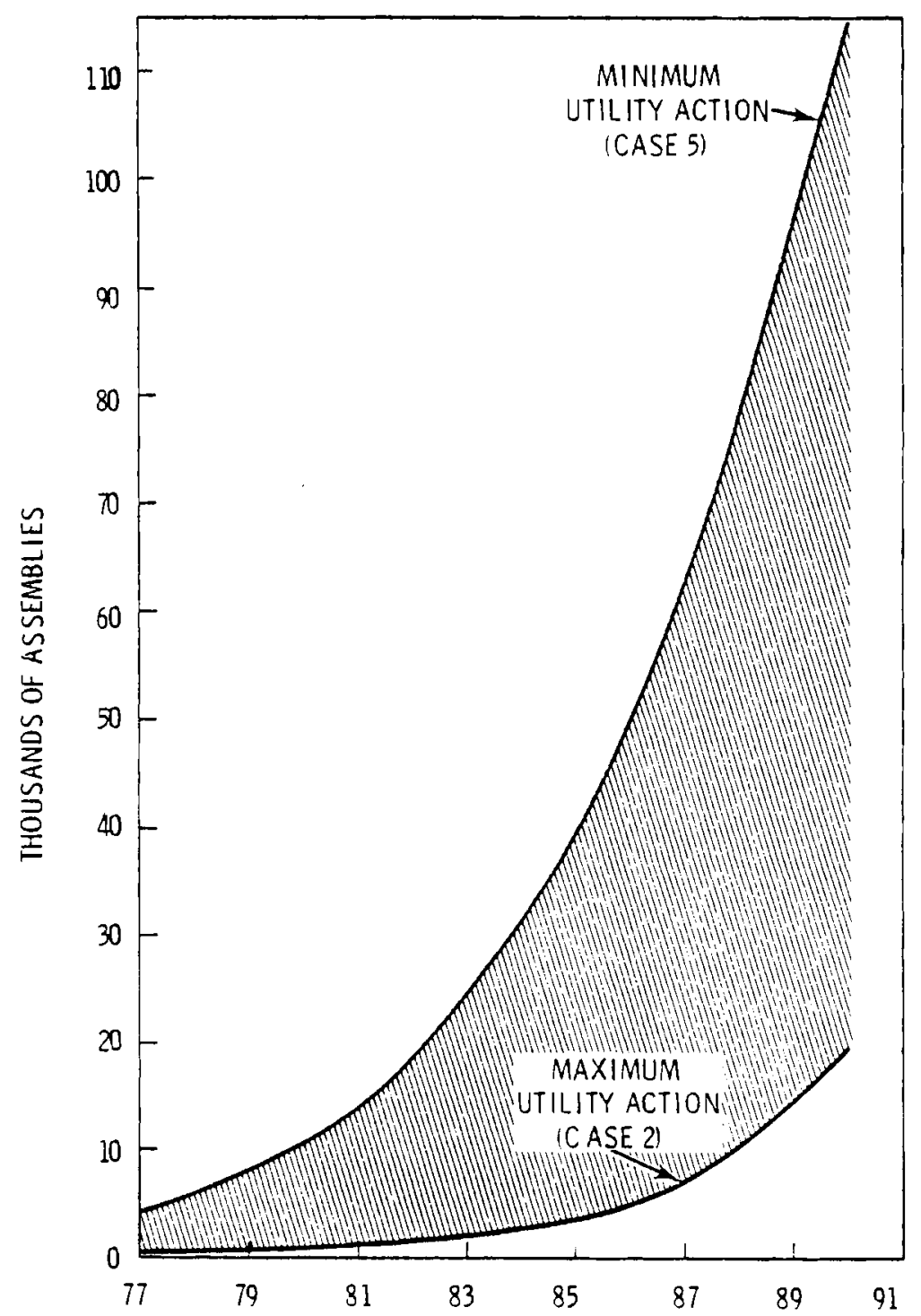

FIGURE 1. Range of Spent Fuei Storage Requirements 
The maximum requirement for additional spent fuel storage capacity occurs if none of the possible industry actions are taken. That is, only those reactor basins for which permission has already been sought from NRC are reracked, full core discharge capability is maintained at every reactor, and no intra-utility fuel transfers are made. For this scenario, additional storage capacity is required for over 23,000 BWR and 17,000 PWR assemblies before 1985 and over 63,000 BWR and 40,000 PWR assemblies by 1990. This case is shown in Figure 1.

The amount of spent fuel which would be shipped to a post reactor bas in storage facility between 1985 and 1990 depends on whether utilities continue the activities and policies required to meet spent fuel storage requirements before 1985 (no full core reserve, intra-utility fuel shipments) or seek to regain their pre-storage shortage posture (full core reserve capacity, no intra-utility fuel shipments). If utilities take the former approach, storage capacity is required between 1985 and 1990 for approximately 11,000 BWR assemblies and 5,000 PWR assemblies. If the latter philosophy prevails, storage capacity must be provided between 1895 and 1990 for approximately 27,000 BWR assemblies and 23,000 PWR assemblies.

\section{DISCUSSION}

The post-reactor basin storage requirements between now and 1990 were calculated for eight scenarios representing variations in reactor basin capacity, intra-utility fuel transfers, and full core reserve policy. These three parameters were varied as follows:

\section{1) Reactor Basin Capacity}

Two levels of reactor basin capacity were considered. The lower level assessed was the present capacity (or planned capacity for reactors yet to be built) augmented by expansion for which licensing requests have been made. The higher level considered assumed that all reactor basins for which no specific expansion plans were known will be expanded to hold three complete cores. 


\section{2) Fu11 Core Reserve}

For the scenarios where full core reserve was assumed, each reactor maintained the capability to discharge an entire core at all times. For reactors sharing a basin, only one full core reserve was assumed in the basin. The cases which assumed no full core reserve projected shipment of fuel offsite only when the reactor basin was completely filled.

3) Intra-Utility Fuel Transfer (IUFT)

For the scenarios which assumed IUFT, it was assumed that spent fuel would be transferred from a full basin to another basin having excess storage capacity at a reactor of the same type (PWR/BWR) within the utility. Basins at new reactors were deemed able to receive transferred spent fuel the year they were projected to enter the utility grid. Varying these three parameters in the manner shown in Figure 2 leads to the eight scenarios evaluated. Figure 2 also lists the major results for each case. Cases 1 through 8 , given in Table 1, give the detailed results of these analyses. The annuai incremental requirement for post reactor basin storage is shown, as well as the cumulative spent fuel storage capacity requirement. The 1985 cumulative capacity calculated for each case identifies the additional spent fuel storage capacity required prior to the achievable availability of any storage capacity other than water basins. The 1985-1990 total capacity requirement identifies potential utilization of a spent fuel storage facility available in 1985 resulting from the case assumptions.

The minimum spent fuel storage capacity requirement from 1985 to 1990 occurs when the actions which must be taken to meet storage requirements before 1985 are continued. This implies that all reactor basins have had their storage capacity increased to accommodate additional spent fuel inventories, that a full core discharge capability is not being maintained, that most operating reactors have full basins due to intra-utility fuel transfer, and some additional AFR storage is in use. The storage capacity increases required between 1985 and 1990 as a result of continuing these actions and policies are shown in Case 2. If utilities desire to discontinue interbasin shipments as soon as possible, the spent fuel storage facility 


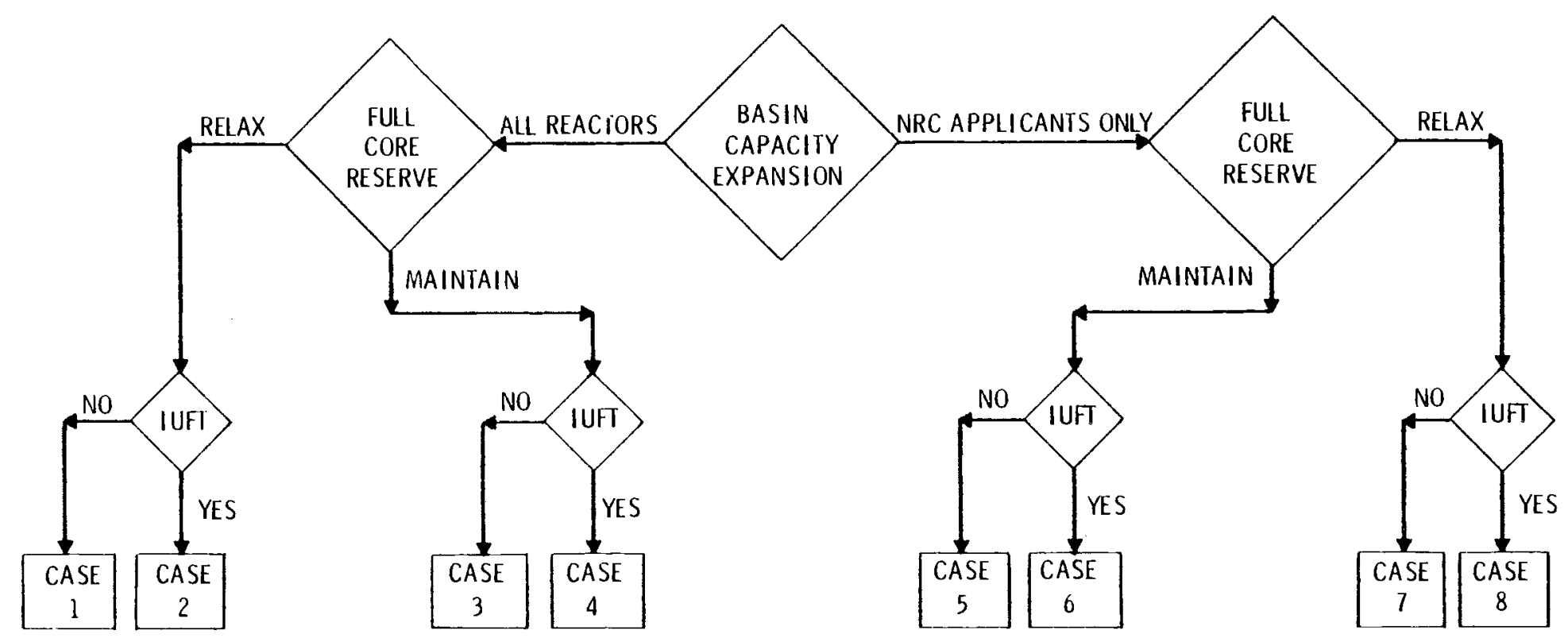

1985 STORAGE REQUIREMENTS (NUMBER OF ASSEMBLIES)

$\begin{array}{lllrl}\text { BWR } & 1235 & 1131 & 6238 & 6238 \\ \text { PWR } & \frac{3615}{4850} & \frac{2033}{3164} & \frac{7715}{13953} & \frac{4394}{10632} \\ \text { TOTAL } & 4850 & 3106 & \end{array}$

1990 STORAGE REQUIREMENIS (NUMBER OF ASSEMBLIES)

$\begin{array}{lllll}\text { BWR } & 12880 & 12239 & 26956 & 24594 \\ \text { PWR } & \underline{13072} & \underline{7239} & \underline{22988} & -19065 \\ \text { TOTAL } & \mathbf{2 5 9 5 2} & \underline{19478} & & \end{array}$

$\begin{array}{llrl}23289 & 18323 & 7866 & 4779 \\ \frac{16990}{40279} & \frac{13024}{31527} & \frac{9457}{17323} & \frac{4315}{9094} \\ & & & \\ 63816 & 53515 & 35408 & 18224 \\ \frac{46542}{110358} & \frac{37376}{90891} & \frac{32281}{67689} & \frac{20654}{38878}\end{array}$

FIGURE 2. Scenario Logic Diagram 


\title{
TABLE 1. Details of the Eight Scenarios Evaluated
}

\author{
CASE 1
}

a) Full Core Reserve Not Ma intained

b) All Reactors increase 3asin Capacity

c) No Intra-utility Fuel Transfer

AFR/STORAGE REQUIREMENTS (ASSEMBLIES)

\begin{tabular}{|c|c|c|c|c|c|c|c|c|c|c|c|c|c|c|c|}
\hline & & 77 & 78 & 79 & 30 & 81 & 82 & 83 & 84 & 85 & 86 & 87 & 88 & 89 & 90 \\
\hline BWR & $\begin{array}{l}\text { Annual } \\
\text { Cumilative } \\
\text { Annual } \\
\text { Cumulative }\end{array}$ & $\begin{array}{r}210 \\
210 \\
38 \\
38\end{array}$ & $\begin{array}{r}62 \\
272 \\
90 \\
128\end{array}$ & $\begin{array}{r}93 \\
365 \\
82 \\
210\end{array}$ & $\begin{array}{r}93 \\
458 \\
203 \\
413\end{array}$ & $\begin{array}{r}93 \\
551 \\
280 \\
693\end{array}$ & $\begin{array}{r}93 \\
644 \\
331 \\
1024\end{array}$ & $\begin{array}{r}93 \\
737 \\
603 \\
1627\end{array}$ & $\begin{array}{r}117 \\
854 \\
987 \\
2614\end{array}$ & $\begin{array}{r}381 \\
1235 \\
1001 \\
3615\end{array}$ & $\begin{array}{r}988 \\
2223 \\
1355 \\
4970\end{array}$ & $\begin{array}{l}1433 \\
3656 \\
1672 \\
6642\end{array}$ & $\begin{array}{l}1914 \\
5570 \\
1982 \\
8624\end{array}$ & $\begin{array}{r}3557 \\
9127 \\
2161 \\
10785\end{array}$ & $\begin{array}{r}3753 \\
12880 \\
2287 \\
13072\end{array}$ \\
\hline
\end{tabular}

3WR Storage Requirement $(1986-1990)=11645$ Assemblies

PWR Storage Reguirement (1986-1990) - 9457 Assemblies

CASE 2

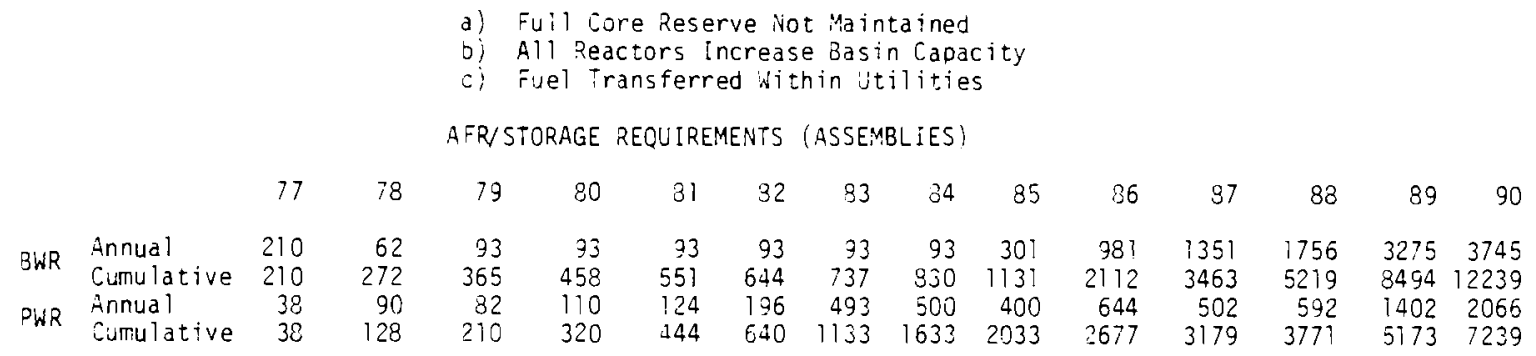

3WR Storage Requirement $(1326-: 990)=11108$ Assemblies

PWR Storage Requirement $(1986-1990)=5206$ Assemblies

CASE 3

a) Full Core Reserve Maintained

b) Ail Reactors Increase Basin Capacity

c) No Intra-utility Fuel iransfer

AFR/STORAGE REQUIREMENTS (ASSEMBLIES)

\begin{tabular}{|c|c|c|c|c|c|c|c|c|c|c|c|c|c|c|c|}
\hline & & 77 & 78 & 79 & 30 & 81 & 32 & 83 & 94 & 85 & 36 & 87 & 88 & 89 & 90 \\
\hline BWR & $\begin{array}{l}\text { Annual } \\
\text { Cumulative } \\
\text { Annual } \\
\text { Cumulative }\end{array}$ & $\begin{array}{l}416 \\
416 \\
401 \\
401\end{array}$ & $\begin{array}{r}93 \\
509 \\
220 \\
627\end{array}$ & $\begin{array}{l}282 \\
791 \\
328 \\
949\end{array}$ & $\begin{array}{r}301 \\
1092 \\
493 \\
1442\end{array}$ & $\begin{array}{r}373 \\
1465 \\
730 \\
2172\end{array}$ & $\begin{array}{r}731 \\
2246 \\
967 \\
3133\end{array}$ & $\begin{array}{r}320 \\
3076 \\
1223 \\
4356\end{array}$ & $\begin{array}{l}1439 \\
4515 \\
1548 \\
5904\end{array}$ & $\begin{array}{l}1723 \\
6238 \\
1811 \\
7715\end{array}$ & $\begin{array}{l}2079 \\
9317 \\
2120 \\
9835\end{array}$ & $\begin{array}{r}3419 \\
11736 \\
2401 \\
12236\end{array}$ & $\begin{array}{r}4198 \\
15934 \\
2853 \\
15089\end{array}$ & $\begin{array}{r}5414 \\
21348 \\
3567 \\
18656\end{array}$ & $\begin{array}{r}5008 \\
26956 \\
4332 \\
22988\end{array}$ \\
\hline & & & $P W R$ & & $\begin{array}{l}\text { Requi } \\
\text { Regui }\end{array}$ & & & $\begin{array}{l}990) \\
990)\end{array}$ & $\begin{array}{l}20713 \\
15273\end{array}$ & & & & & & \\
\hline
\end{tabular}

CASE 4

a) Full Core Reserve Maintained

b) A11 Reactors Increase Basin Capacity

c) Fuel Transferred :Iithin Utilities

AFR/STORAGE REQUIREMENTS (ASSEMBLIES)

\begin{tabular}{|c|c|c|c|c|c|c|c|c|c|c|c|c|c|c|}
\hline & 77 & 78 & 79 & 30 & 81 & 32 & 83 & 84 & 85 & 86 & 87 & 88 & 39 & \\
\hline $\begin{array}{l}\text { Annual } \\
\text { Cumulative } \\
\text { Annual } \\
\text { Cumulative }\end{array}$ & $\begin{array}{l}416 \\
416 \\
195 \\
195\end{array}$ & $\begin{array}{r}93 \\
509 \\
246 \\
447\end{array}$ & $\begin{array}{r}93 \\
602 \\
203 \\
644\end{array}$ & $\begin{array}{r}93 \\
695 \\
381 \\
1025\end{array}$ & $\begin{array}{r}153 \\
348 \\
456 \\
1481\end{array}$ & $\begin{array}{r}765 \\
1613 \\
479 \\
1960\end{array}$ & $\begin{array}{r}1347 \\
2960 \\
586 \\
2546\end{array}$ & $\begin{array}{r}1555 \\
4515 \\
804 \\
3350\end{array}$ & $\begin{array}{l}1723 \\
6238 \\
1044 \\
4394\end{array}$ & $\begin{array}{l}1811 \\
8049 \\
1451 \\
5845\end{array}$ & $\begin{array}{r}3159 \\
11208 \\
2403 \\
3248\end{array}$ & $\begin{array}{r}3289 \\
14497 \\
2686 \\
10934\end{array}$ & $\begin{array}{r}4315 \\
18812 \\
3783 \\
14717\end{array}$ & $\begin{array}{r}5783 \\
24595 \\
4348 \\
19065\end{array}$ \\
\hline
\end{tabular}

BWR Storage Requirement $(1986-1990)=18357$ Assemblies

PWR Storage Requirement (1986-1990) - 15273 Assemblies 


\section{TABLE 1. (cont'd)}

CASE 5

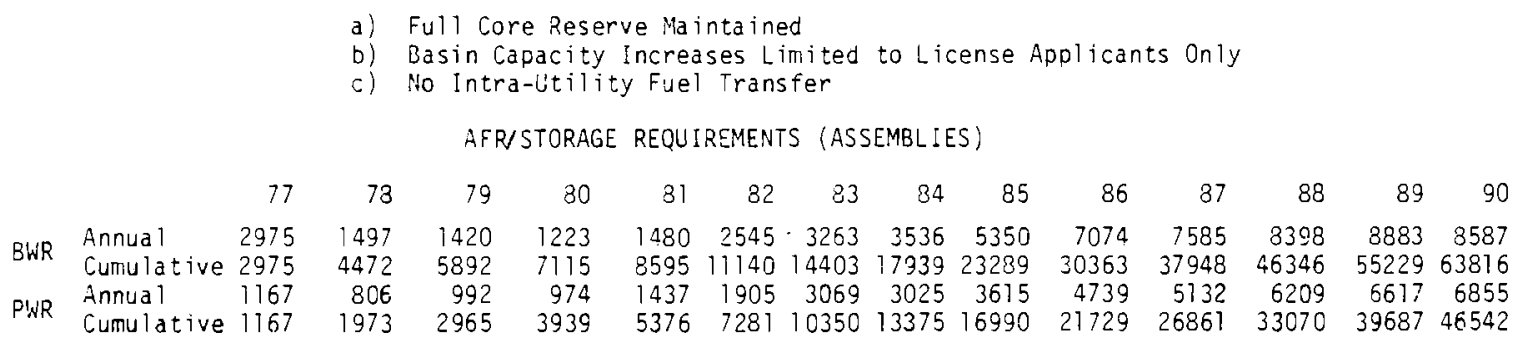

BWR Storace Requirement $(1986-1990)=40527$ Assembl ies

PHIR Storage Requirement $(1986-1990)=29552$ Assemblies

CASE 6

a) Full Core Reserve Maintained

b) Basin Capacity Increases Limited to License Apolicants Only

c) Fuel Transferred Within Utilities

AFR/STORAGE REQUIREMENTS (ASSEMBLIES)

\begin{tabular}{|c|c|c|c|c|c|c|c|c|c|c|c|c|c|c|c|}
\hline & & 77 & 73 & 79 & 80 & 31 & 2 & 83 & 84 & 85 & 36 & 87 & 88 & 29 & \\
\hline & & & & $\begin{array}{r}522 \\
3762\end{array}$ & $\begin{array}{r}389 \\
4157\end{array}$ & & & & & & & & & & \\
\hline & $\begin{array}{l}\text { Annua } 1 \\
\text { Cumulative }\end{array}$ & $\begin{array}{l}671 \\
671\end{array}$ & $\begin{array}{r}462 \\
1133\end{array}$ & $\begin{array}{r}547 \\
1630\end{array}$ & $\begin{array}{r}794 \\
2474\end{array}$ & $\begin{array}{l}1041 \\
3515\end{array}$ & $\begin{array}{l}1268 \\
4783\end{array}$ & $\begin{array}{l}1355 \\
5638\end{array}$ & $\begin{array}{l}3020 \\
9658\end{array}$ & & & & & $\begin{array}{r}5507 \\
31224\end{array}$ & \\
\hline
\end{tabular}

3WR Storage Requirement $(1936-1990)=35192$ Assemblies

PIJR Storage Requirement $(1986-1990)=24352$ Assemblies

CASE 7

a) Fu11 Core Reserve lot Maintained

b) Basin Capacity Increases Limited to License Applicants Oniy

c) No Intra-Utility Fuel Transfer

AFR/STORAGE REQULREMENTS (ASSEMBLIES)

\begin{tabular}{|c|c|c|c|c|c|c|c|c|c|c|c|c|c|c|}
\hline & 77 & 78 & 79 & 80 & 81 & 32 & 03 & 84 & 85 & 86 & 87 & 38 & 39 & 90 \\
\hline $\begin{array}{l}\text { Annual } \\
\text { Cumul a tive } \\
\text { Annual }\end{array}$ & $\begin{array}{l}216 \\
216 \\
144\end{array}$ & $\begin{array}{l}180 \\
396 \\
220\end{array}$ & $\begin{array}{l}330 \\
726 \\
418\end{array}$ & $\begin{array}{l}212 \\
938 \\
355\end{array}$ & $\begin{array}{r}234 \\
1222 \\
842\end{array}$ & $\begin{array}{r}646 \\
1868 \\
987\end{array}$ & $\begin{array}{l}1713 \\
3531 \\
1649\end{array}$ & $\begin{array}{l}1538 \\
5169 \\
2371\end{array}$ & $\begin{array}{l}2697 \\
7866 \\
2471\end{array}$ & $\begin{array}{r}3337 \\
11203 \\
2923\end{array}$ & $\begin{array}{r}3906 \\
15109 \\
3520\end{array}$ & $\begin{array}{r}5234 \\
20343 \\
4639\end{array}$ & $\begin{array}{r}7286 \\
27629 \\
5380\end{array}$ & $\begin{array}{r}354 \\
63\end{array}$ \\
\hline
\end{tabular}

BWR Storage Requirement $(1986-1990)=27542$ Assemblies

PuR Storare Requirement $(1986-1990)=22824$ As semblies

CASE \&

a) Full Core Reserve Not Maintained

b) Basin Capacity Increases Limited to License Applicants Only

c) Fuel Transferred $\forall i$ thin Utiltities

AFR/STORAGE REQUIREMENTS (ASSEMBLIES)

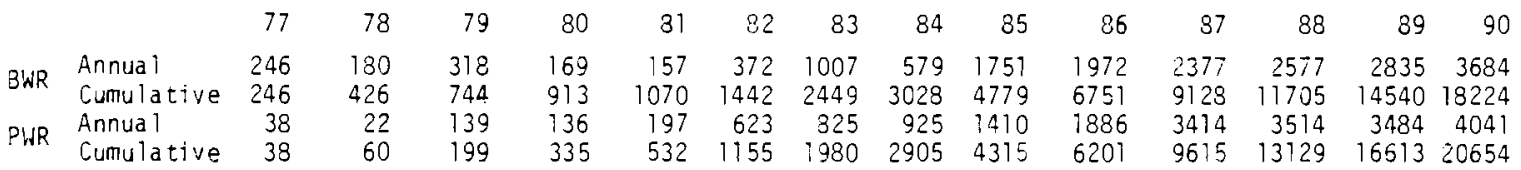

BWR Storage Requirement $(1986-1990)=13445$ Assembl ies P.SR Storage Requirement $(1986-1990)=16339$ Assemblies 
capacity requirement is equal to the reactor discharge rate at the time the storage facility starts operation. If utilities further desire to reestablish FCR within 3 or 4 years, the required spent fuel facility storage capacity is doubled.

This process of progressing from the existing spent fuel situation to a more desirable situation may be viewed as a transition from case 2 to case 3 . This is shown graphically for BWRs in Figure 3 . The spent fuel storage facility receipt rate to effect this transition is approximately 5,400 BWR assemblies per year, or about 27,000 BWR assemblies from 1985-1990. The PWR spent fuel storage requirements are approximately 4600 assemblies per year, or 23,000 assemblies from 1985 to 1990.

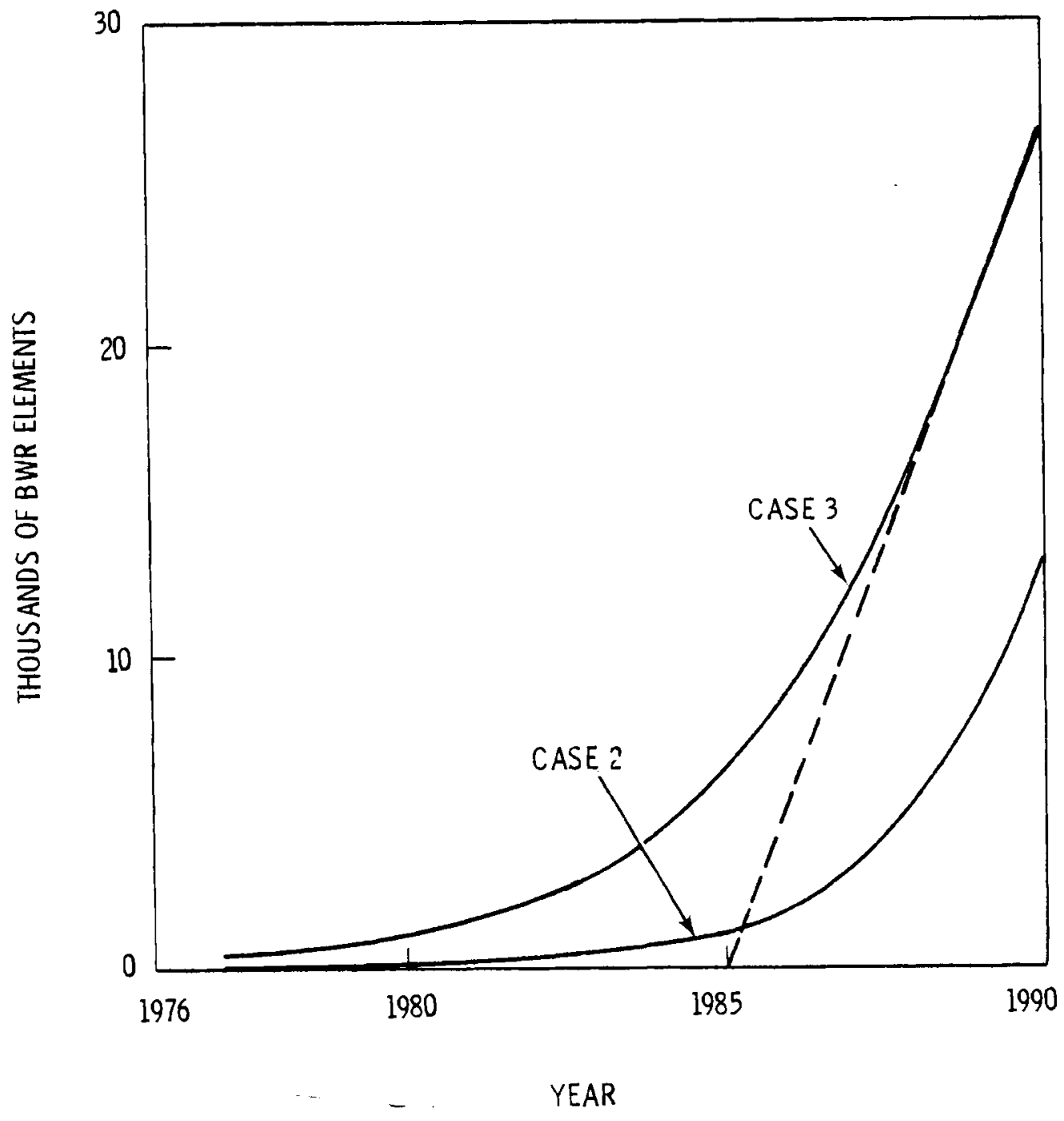

FIGURE 3. BWR Spent Fuel Storage Requirements 


\section{DATA BASE}

The Appendix 1 ists the reactors for which spent fue 1 storage requirements were assessed, their type (BWR/PWR), power generation capacity, and operating data. Data for reactor discharges, startup dates, basin capacities, and current basin inventories were obtained from Y/OWI/SUB-77/42500, prepared by Nuclear Assurance Corporation for the Office of Waste Isolation. This report includes reactors which will be operational by 1986 . For reactors which were projected to become operational from 1986 through 1990, projected discharges were modeled on those in the OWI report of the same reactor size and design. For the cases that limited basin capacity increases to license applicants only, reactors for which no basin expansion license had been requested were assumed to have the basin capacities specified in their respective Safety Analys is Reports.

\section{REFERENCES}

1. LWR Spent Fuel Disposition Capabilities 1977-1986. ERDA 77-25, Energy Research and Development Administration, 1977 Edition. Available from NTIS.

2. Testimony of L. V. Gossick before the House Subcommittee on Oversight, Investigation of the Committee on Interstate and Foreign Commerce, August $1,1977, \underline{23}(32): 2$, August 8, 1977.

3. U.S. LWR Spent Fuel Inventory and Projection, Y/OWI/SUB-77/42500, Nuclear Assurance Corporation, Atlanta, GA, June 1977. 
. 
APPENDIX

REACTOR DATA BASE 
REACTOR DATA BASE

Alabama Power Company

\begin{tabular}{lccc} 
Reactor & Type & $\begin{array}{c}\text { Generating } \\
\text { Capacity (MWe) }\end{array}$ & Operating Date \\
\cline { 3 - 4 } Farley 1 & PWR & 861 & 1977 \\
Farley 2 & PWR & 867 & 1979 \\
Barton 2 & BWR & 1300 & 1986 \\
Barton 2 & BWR & 1300 & 1987 \\
Arizona Public Service Company & & \\
\hline Palo Verde 2 & PWR & & 1982 \\
Palo Verde 2 & PWR & 1237 & 1984 \\
Palo Verde 3 & PWR & 1237 & 1985
\end{tabular}

Arkansas Power and Light Company

$\begin{array}{lllr}\text { Arkansas 1* } & \text { PWR } & 886 & \text { Dec. } 1974 \\ \text { Arkansas 2 } & \text { PWR } & 960 & \\ 1978\end{array}$

Baltimore Gas and Electric Company

$\begin{array}{lrrr}\text { Calvert Cliffs 1 } & \text { PWR } & 880 & \text { May } 1975 \\ \text { Calvert Cliffs 2 } & \text { PWR } & 880 & 1977 \\ \text { Boston Edison Company } & & & \\ \text { Pilgrim 1* } & & & \\ \text { Pilgrim 2 } & \text { BWR } & 685 & \text { Dec. } 1972 \\ \text { PWR } & 1770 & & 1984\end{array}$

Carolina Power and Light Company

$\begin{array}{llrr}\text { Robinson 2* } & \text { PWR } & 739 & \text { Apri1 } 1971 \\ \text { Brunswick 2* } & \text { BWR } & 849 & \text { Dec. } 1975 \\ \text { Brunswick 1* } & \text { BWR } & 849 & 1977 \\ \text { Harris 1 } & \text { PWR } & 930 & 1983 \\ \text { Harris 2 } & \text { PWR } & 930 & 1985 \\ \text { Harris 3 } & \text { PWR } & 930 & 1989 \\ \text { Harris 4 } & \text { PWR } & 930 & 1987\end{array}$

Cincinnati Gas and Electric Company

$\begin{array}{lrrr}\text { Zirmer 1 } & \text { BWR } & 840 & 1979 \\ \text { Zimmer 2 } & \text { BWR } & 1172 & 1986\end{array}$

Cleveland Electric 11 luminating Company

$\begin{array}{llll}\text { Perry } 1 & \text { BWR } & 1300 & 1981 \\ \text { Perry } 2 & \text { BWR } & 1300 & 1983\end{array}$

*Applications to expand spent fuel storage capacity 
Commonwealth Edison Company

\begin{tabular}{|c|c|c|c|}
\hline Reactor & Type & $\begin{array}{c}\text { Generating } \\
\text { Capacity (MWe) }\end{array}$ & Operating Date \\
\hline $\begin{array}{l}\text { Dresden 1 } \\
\text { Dresden 2* } \\
\text { Dresden 3* } \\
\text { Quad City 1* } \\
\text { Quad City 2*} \\
\text { Zion 1* } \\
\text { Zion 2* } \\
\text { LaSalle } 1 \\
\text { LaSalle } 2 \\
\text { Byron 1 } \\
\text { Bryon } 2 \\
\text { Braidwood } 1 \\
\text { Braidwood } 2 \\
\text { Carroll 1 } \\
\text { Carroll } 2\end{array}$ & $\begin{array}{l}\text { BWR } \\
\text { BWR } \\
\text { BWR } \\
\text { BWR } \\
\text { BWR } \\
\text { PWR } \\
\text { PWR } \\
\text { BWR } \\
\text { BWR } \\
\text { PWR } \\
\text { PWR } \\
\text { PWR } \\
\text { PWR } \\
\text { PWR } \\
\text { PWR }\end{array}$ & $\begin{array}{r}215 \\
834 \\
832 \\
832 \\
832 \\
1085 \\
1085 \\
1122 \\
1122 \\
1118 \\
1118 \\
1118 \\
1118 \\
1150 \\
1150\end{array}$ & $\begin{array}{r}\text { Aug. } 1960 \\
\text { Aug. } 1972 \\
\text { Sept. } 1971 \\
\text { Feb. } 1973 \\
\text { Mar. } 1973 \\
\text { Dec. } 1973 \\
\text { Sept. } 1974 \\
1979 \\
1980 \\
1980 \\
1982 \\
1981 \\
1982 \\
1986 \\
1988\end{array}$ \\
\hline
\end{tabular}

Connecticut Yankee Atomic Power Company

Haddam Neck* PWR

PWR

600

Jan. 1968

Consolidated Edison Company

Indian Pt. ? PWR

Indian Pt. 2*

Indian Pt. $3^{\star}$

PWR

PWR

Consumers Power Company

Big Rock Pt.

Pal isades*

Midland 1

Midland 2

\section{BWR}

PWR

PWR

PWR

Detroit Edison Company

Fermi 2

Greenwood 2

Greenwood 3

BWR
PWR

PWR

1150

1300

1300

Duke Power Company

Oconee 1

Oconee 2

Oconee $3^{*}$

McGuire 1

McGuire 2

Catawba 1

Catawba 2

Perkins 1

Perkins 2

Cherokee 1

Cherokee 2

Perkins 3

Cherokee 3

$\begin{array}{lr}\text { PWR } & 911 \\ \text { PWR } & 911 \\ \text { PWR } & 911 \\ \text { PWR } & 1180 \\ \text { PWR } & 1180 \\ \text { PWR } & 1150 \\ \text { PWR } & 1300 \\ \text { PIR } & 1300 \\ \text { PWR } & 1300 \\ \text { PWR } & 1300 \\ \text { PWR } & 1300 \\ \text { PWR } & 1300 \\ \text { PWR } & 1300\end{array}$

July 1973

Sept 1974

Dec. 1974

1979

1980

1981

1985

1985

1987

1984

1989

1989

1987

ॠApplications to expand spent fuel storage capacity 
Duquesne Light Company

\begin{tabular}{|c|c|c|c|}
\hline Reactor & Type & $\begin{array}{c}\text { Generating } \\
\text { Capacity (MWe) }\end{array}$ & Operating Date \\
\hline $\begin{array}{l}\text { Beaver Valley } 1^{*} \\
\text { Beaver Valley } 2\end{array}$ & $\begin{array}{l}\text { PWR } \\
\text { PWR }\end{array}$ & $\begin{array}{l}923 \\
888\end{array}$ & $\begin{array}{ll}\text { Sept. } & 1976 \\
1982\end{array}$ \\
\hline
\end{tabular}

Florida Power and Light Company

Turkey Pt. $3^{*} \quad$ PWR

Turkey Pt. 4* PWR 728

st. Lucie 2

PWR

PWR

Florida Power Corporation

Crystal River 3

PWR

825

Georgia Power Company

Hatch 1

Hatch 2

BWR

$B W R$

813

813

980

River Bend 1

River Bend 2

Blue Hills 1

Blue Hills 2

BWR

BWR

PWR

PWR

Houston Lighting and Power Company

Allens Creek 1

Allens Creek 2

South Texas 1

South Texas 2

\section{BWR}

BWR

PWR

PWR

Illinois Power Company

Clinton 1

Clinton 2

BWR

BWR

Indiana and Michigan Electric Company

D. C. Cook 1

D. C. CoOk 2

PWR

PWR

1090

1090

Iowa Electric Light and Power Company

\begin{tabular}{|c|c|c|}
\hline $\begin{array}{l}\text { D. Arnold 1* } \\
\text { Central Iowa }\end{array}$ & $\begin{array}{l}\text { BWR } \\
\text { PWR }\end{array}$ & $\begin{array}{r}565 \\
1200\end{array}$ \\
\hline the & -1911 & \\
\hline $\begin{array}{l}\text { Oyster Creek* } \\
\text { Forked River }\end{array}$ & $\begin{array}{l}\text { BW'R } \\
\text { PWR }\end{array}$ & $\begin{array}{r}65 \\
117\end{array}$ \\
\hline
\end{tabular}

*Applications to expand spent fuel storage capacity
1977

Aug. 7975

Dec. 1972

Sept. 1973

June 1976

1981

Jan. 1976

1979

1982

1984

1985

1987

1985

1986

1980

1981

1980

7984

980

1978

Feb. 1975

1987

Dec. 1969

1983 
Kansas Utilities

\begin{tabular}{|c|c|c|c|}
\hline Reactor & Type & $\begin{array}{c}\text { Generating } \\
\text { Capacity (MNe) }\end{array}$ & Operating Date \\
\hline Wolf Creek & PWR & 1150 & 1983 \\
\hline \multicolumn{4}{|c|}{ Louisiana Power and Light Company } \\
\hline Waterford 3 & PWR & 980 & 1981 \\
\hline \multicolumn{4}{|c|}{ Long Island Lighting Company } \\
\hline $\begin{array}{l}\text { Shoreham } \\
\text { Jamesport } 1 \\
\text { Jamesport } 2\end{array}$ & $\begin{array}{l}\text { BWR } \\
\text { PWR } \\
\text { PWR }\end{array}$ & $\begin{array}{r}849 \\
1150 \\
1150\end{array}$ & $\begin{array}{l}1978 \\
1984 \\
1986\end{array}$ \\
\hline
\end{tabular}

Maine Yankee Atomic Power Company

Maine Yankee* PWR

Metropolitan Edison Company

Three Mile Is. 1. PWR

Three Mile Is. 2 PWR

Mississippi Power and Light Company

Grand Gulf 1

BWR

1300

Grand Gulf 2

BWR

1300

1981

Nebraska Public Power District

Cooper BWR

Nebraska ?

BWR
PL'R

800

1000

June 1974

1984

Niagra Mohawk Power Company

Nine Mile Pt. 1*

Nine Mile Pt. 2

BWR
BWR

610

1150

Dec. 1969

1982

Northern Indiana Public Service Company

Bailly

BWR

685

1982

Northeast Nuclear Energy Company

$\begin{array}{llr}\text { Millstone } 1^{*} & \text { BWR } & 682 \\ \text { Milistone 2* } & \text { PWR } & 365 \\ \text { Millstone 3 } & \text { PWR } & 1124 \\ \text { Montague 1 } & \text { BWR } & 1200 \\ \text { Montague 2 } & \text { BWR } & 1200\end{array}$

Jan. 1972

Dec. 1975

1982

1987

Montague

BWR

1200

1989

FApplications to expand spent fuel storage capacity 
Northern States Power Company

\begin{tabular}{lccc} 
Reactor & Type & $\begin{array}{c}\text { Generating } \\
\text { Capacity (MWe) }\end{array}$ & Operating Date \\
\cline { 3 - 4 } & BWR & 580 & JuTy 1971 \\
Monticel10 & PWR & 547 & Dec. 1973 \\
Prairie Is. 1* & PWR & 547 & Dec. 1974 \\
Prairie Is. 2* & PWR & 1150 &
\end{tabular}

Omaha Public Power District

Fort Calhoun 1* PWR

Fort Calhoun 2 PWR

481

Sept. 1973

$1150 \quad 1983$

Power Authority of the State of New York

Fitzpatrick

BWR

821

Ju 1 y 1975

Pennsylvania Power and Light Company

Susquehana 1

$\begin{array}{ll}\text { BWR } & 1100\end{array}$

Susquehana 2

BWR

1100

1982

Pacific Gas and Electric Company

Humbolt Bay

BWR

PWR

PWR

BWR

BWR

Diablo Canyon

1200

Stanislous 2

1200

Aug. 1962

1977

1977

1989

1990

Philadelphia Electric Company

Peach Bottom 2

SWR $\quad 1098$

1098

1100
1100

Limerick ?

BWR

BWR

May 1974

Dec. 1974

1983

Limerick 2

BWR

1985

Portland General Electric

$\begin{array}{lll}\text { Trojan* } & \text { PWR } & 1216 \\ \text { Pebble Springs 1 } & \text { PWR } & 1300\end{array}$

Pebble Sorings 2

PWR

1200

May 1976

1985

1987

Public Service Company of Indiana

Marble Hill

PWR

1150

1981

Marble Hill 2

PWR

1150

1983

Public Service Company of Oka lahoma

Black Fox 1

BWR

980

1983

Black Fox 2

BWR

980

1985

*Applications to expand spent fuel storage capacity 
Public Service Electric and Gas Company of New Jersey

\begin{tabular}{lccr} 
Reactor & Type & $\begin{array}{c}\text { Generating } \\
\text { Capacity (MWe) }\end{array}$ & Operating Date \\
\cline { 1 - 1 } Salem 1* & PWR & 1170 & June 1977 \\
Salem 2 & PWR & 1170 & 1979 \\
Hope Creek 1 & BWR & 1140 & 1984 \\
Atlantic 1 & PWR & 1150 & 1984 \\
Hope Creek 2 & BWR & 1150 & 1985 \\
Atlantic 2 & PWR & 1150 & 1985
\end{tabular}

Public Service Company of New Hampshire

Seabrook 1

Seabrook 2

PWR

PWR

1150

1150

Puget Sound Power and Light Company

Skagit 1

BWR

1300

1984

Rochester Gas and Electric Company

R. E. Ginna*

Sterling 1

PWR

PWR

490

1150

South Carolina Electric and Gas Company

V. Surmer ?

PWR

950

Southern California Edison Company

San Onofre 1

San Onofre 2

PWR

PWR

PWR

456

1170

1170

Sacramen to Municpal Utility District

Rancho Seco 1*

Rancho Seco 2

PWR

PWR

San Diego Gas and Electric Company

Sun Desert 1

Sun Desert 2

PWR

PWR

790

790

Texas Utilities

Commanche Peak 1

Commanche Peak 2

PWR

PWR

1150

1150

Toledo Edison Company

Davis Besse

Davis Besse 2

PWR

PWR

950

950

Davis Besse 3

PWR
1985

1988

1980

Mar. 1970

1984

1980

Jan. 1968

1981

1983

Apr. 1975

1987

1982

June 1977

1985

1986

\#Applications to expand spent fuel storage capacity 
Tennessee Valley Authority

\begin{tabular}{ll}
\multicolumn{1}{c}{ Reactor } & Type \\
Brown's Ferry 1 & BWR \\
Brown's Ferry 2 & BWR \\
Brown's Ferry 3 & BWR \\
Sequoyan 1 & PWR \\
Sequoyah 2 & PWR \\
Watts Bar 1 & PWR \\
Watts Bar 2 & PWR \\
Bellefonte 1 & PWR \\
Bellefonte 2 & PWR \\
Hartsville A-1 & BWR \\
Hartsville A-2 & BWR \\
Hartsville B-1 & BWR \\
Hartsville B-2 & BWR \\
Yellow Creek 1 & PWR \\
Yellow Creek 2 & PWR \\
Union Electric Company of St. Louis
\end{tabular}

\begin{tabular}{|c|c|}
\hline $\begin{array}{c}\text { Generating } \\
\text { Capacity (Mwe) } \\
\end{array}$ & Operating Date \\
\hline $\begin{array}{l}1098 \\
1098 \\
1098 \\
1170 \\
1170 \\
1218 \\
1218 \\
1300 \\
1218 \\
1300 \\
1300 \\
1300 \\
1300 \\
1300 \\
1300\end{array}$ & $\begin{array}{c}\text { Aug. } 1974 \\
\text { Mar. } 1975 \\
1977 \\
1978 \\
1979 \\
1979 \\
1980 \\
1980 \\
1981 \\
1983 \\
1984 \\
1983 \\
1984 \\
1985 \\
1988\end{array}$ \\
\hline $\begin{array}{l}1150 \\
1150\end{array}$ & $\begin{array}{l}1982 \\
1984\end{array}$ \\
\hline
\end{tabular}

$\begin{array}{llll}\text { Callaway } 1 & \text { PWR } & 1150 & 1982 \\ \text { Callaway } 2 & \text { PWR } & 1150 & 1984\end{array}$

Virginia Electric and Power Company

$\begin{array}{lllr}\text { Surry 1* } & \text { PWR } & 824 & \text { Dec. } 1972 \\ \text { Surry 2* } & \text { PWR } & 824 & \text { May 1973 } \\ \text { North Anna 1 } & \text { PWR } & 947 & \text { Apr. 1977 } \\ \text { North Anna 2 } & \text { PWR } & 892 & 1978 \\ \text { Surry 3 } & \text { PWR } & 915 & 1987 \\ \text { Surry 4 } & \text { PWR } & 915 & 1988 \\ \text { North Anna 3 } & \text { PWR } & 915 & 1981 \\ \text { North Anna 4 } & \text { PWR } & 915 & 1981\end{array}$

Vermont Yankee Nuclear Power Corporation

Vermont Yankee* BWR

$540 \quad$ Nov. 1972

Washington Public Power Supply System

$\begin{array}{llll}\text { WPPSS } 2 & \text { BWR } & 1150 & 1980 \\ \text { WPPSS } 1 & \text { PWR } & 1300 & 1982 \\ \text { WPPSS } 3 & \text { PWR } & 1240 & 1983 \\ \text { WPPSS } 4 & \text { PWR } & 1300 & 1983 \\ \text { WPPSS } 5 & \text { PWR } & 1240 & 1985\end{array}$

Wisconsin Electric Power Company

$\begin{array}{lllr}\text { Point Beach 1* } & \text { PWR } & 524 & \text { Dec. } 1970 \\ \text { Point Beach 2* } & \text { PWR } & 524 & \text { Oct. } 1972 \\ \text { Koshkonong 1 } & \text { PWR } & 930 & 1986 \\ \text { Koshkonong 2 } & \text { PWR } & 930 & 1989\end{array}$

*Applications to expand spent fuel storage capacity 
Wiscons in Public Service Company

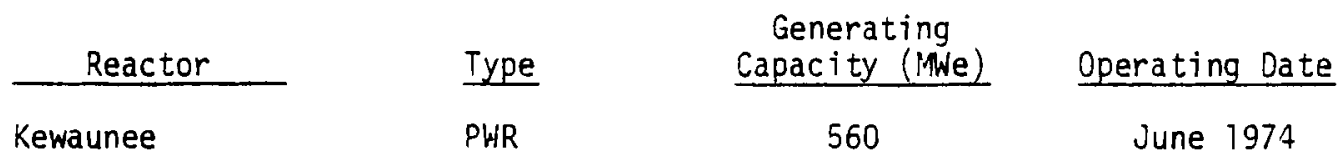

Yankee Atomic Electric Company

Yankee Rowe* PWR

185

June 1961

New York State Electric and Gas Company

Somerset 1

BWR

1300

1300

1984

Somerset 2

BWR

1985

Central Ma in Power Company

Sears Island

PNR

1124

1985

Dairyland Power Company

La Crosse*

BWR

55

Nov. 1969

Ohio Edison Company

Erie 1

Erie 2

$B W R$

1260

1987

*Applications to expand spent fue] storage capacity 
No. of

Copies

OFFSITE

A. A. Churm

DOE Chicago Patent Group

Chicago Operations Office

9800 South Cass Avenue

Argonne, IL 60439

27 DOE Technical Information Center

W. W. Ballard

U.S. Department of Energy Headquarters

Washington, DC 20545

C. R. Cooley

U.S. Department of Energy Headquarters

washington, DC 20545

M. J. Lawrence

U.S. Department of Energy Headquarters

Washington, DC 20545

J. P. Thereault

U.S. Department of Energy Headquarters

Washington, DC 20545

J. B. Work

U.S. Department of Energy Headquarters

Washington, DC 20545

A. Roberts

U.S. Department of Energy Nevada Operations Office

P.0. Box 14100

Las Vegas, NV 89114
No. of

Copies

D. W. Turner

Oak Ridge Nationa 1

Laboratory

P.0. Box X

Oak Ridge, TN 37830

3 F. D. King

Savannah River Laboratory

P.0. Drawer $E$

Aiken, SC 29801

2 D. C. Durrill

Westinghouse Electric Corporation Advanced Energy Systems Division

E. Pittsburg, PA 15112

2 J. B. Wright

Westinghouse Electric Corporation Advanced Energy Systems Division

E. Pittsburg, PA 15112

\section{ONSITE}

U.S. Department of Energy Richland Operations Office

J. C. Cummings

O. J. Elgert

P. G. Harris

F. L. Goldsberry

R. B. Goranson (7)

H. E. Ransom

Pacific Northwest

Laboratory

W. B. Andrews

A. J. Boegel

N. E. Carter

R. L. Engel

R. M. Fleischman

C. M. Heeb

I. S. Levy

R. C. Lijkala 
No. of

Copies

ONSITE

Pacific Northwest

Laboratory (cont'd)

E. T. Merri11

D. F. Newman

M. K. White (10)

R. D. Widrig

L. D. Williams

C. E. Willingham

Technical Information Files (5)

Technical Publications (2)

Rockwe11 Hanford Operations

J. R. Cole

J. M. Davis

R. B. Gerth

R. J. Gimera

K. H. Henry

R. A. Kennedy

W. J. Kurzeka

W. H. Peter

D. D. Ritter

R. C. Roal

D. A. Turner

D. D. Wodrich

Central File

Document File (2)

Hanford Engineering Development Laboratory

R. L. Knecht (2) 